\title{
Dinámica de la producción de maíz y frijol en México de 1980 a $2014^{1}$
}

\author{
Dynamics production of corn and beans in Mexico from 1980 to 2014
}

\author{
Mauricio De los Santos-Ramos ${ }^{2}$, Teolincacihuatl Romero-Rosales ${ }^{2}$,Encarnación Ernesto Bobadilla-Soto ${ }^{2}$
}

\begin{abstract}
Resumen
El objetivo de este trabajo fue evaluar la dinámica de la producción de maíz y frijol en México. Se efectuó un análisis retrospectivo para identificar los puntos críticos, los cambios y ajustes dados en el sector como resultado de las políticas del gobierno mexicano. Los datos fueron obtenidos de SAGARPA, Banco de México, Secretaría de Economía y de la Organización de las Naciones Unidas para la Alimentación y la Agricultura. Se evaluaron indicadores de tasa de crecimiento media anual (TCMA), tendencia y precios constantes. Al realizarse el análisis nacional, se consideró como variable al país en su conjunto. Se analizaron los datos de superficie sembrada, producción, precios e importaciones de maíz y frijol en México entre los años 1980 a 2014. La superficie sembrada de maíz y frijol tuvo un decremento de 1,8 millones y 193 mil hectáreas, respectivamente; el rendimiento se incrementó para el maíz en 1,47 t/ha y en frijol $160 \mathrm{~kg} / \mathrm{ha}$, la producción de maíz tuvo una TCMA de 1,8\% equivalente 10,1 millones de toneladas, el frijol tuvo una TCMA 0,89\% con un incremento de 338783 t. El maíz y frijol a precios constantes han tenido una disminución del 64,6\% y 59,0\%, correspondiente a 441,9 y 976,3 dólares por tonelada, respectivamente. Las importaciones de maíz tuvieron una TCMA de 2,8\%, finalizó el 2012 con 9,5 millones de toneladas, las importaciones de frijol no mostraron ninguna tendencia. El punto crítico en las importaciones se dio con la entrada en vigor del Tratado de Libre Comercio de América del Norte, pasó de una economía cerrada a una abierta, donde se incrementaron más las importaciones que la producción nacional.
\end{abstract}

Palabras claves: importaciones de maíz, rendimiento de cultivos, desempeño de cultivos.

\begin{abstract}
The objective of this work was to evaluate the dynamic of corn and bean production in Mexico. Retrospective analysis was conducted in order to identify critic points, changes and adjustments given in the sector, as a result of Mexican government policies. Data was obtained from SAGARPA, Bank of Mexico, Secretary of Economy and Food and Agriculture Organization. Indicators were evaluated, as annual average growth rate (TCMA) and growing indicators, trends and real prices were evaluated. When doing the analysis, it was considered as a variable the country as a whole. The data of plantings, production, prices and imports of corn and beans in Mexico between 1980 and 2014 was analyzed. The plantings of corn and beans showed a decrease of 1.8 million and 193 thousand hectares respectively, the performance of corn increased on $1.47 \mathrm{t} / \mathrm{ha}$ and for beans, and $160 \mathrm{~kg} / \mathrm{ha}$ for corn production and had a TCMA of $1.8 \%$ equivalent to 10.1 million tons, and beans had a TCMA of $0.89 \%$ with an increase of 338783 t. Corn and beans, at constant prices, had a decrease of $64.6 \%$ and $59.0 \%$, the decreased was of 441.9 and 976.3 dollars per ton respectively.
\end{abstract}

\footnotetext{
Recibido: 31 de marzo, 2016. Aceptado: 5 de julio, 2016. Este trabajo forma parte de las actividades de la Academia de Economía y Administración del Centro de Estudios Profesionales del Colegio Superior Agropecuario del Estado de Guerrero, México.

2 Colegio Superior Agropecuario del Estado de Guerrero, Centro de Estudios Profesionales y Centro de Estudios Técnicos. Iguala, estado de Guerrero, México. C.P. 40000. mauriciodelosantos@yahoo.com.mx, bosee03@hotmail.com (autor para correspondencia), teolinc@hotmail. com
} 
The imports of corn had a TCMA of $2.84 \%$ ending 2012 with 9.5 million tons, the imports of beans don't show any trend. The critic point of imports began with the North American Free Trade Agreement, where Mexico changed from a close economy to an open one, in which the government stimulates more imports, than national production.

Keywords: corn importation, bean importation, crop performance.

\section{Introducción}

Todas las naciones consideran a la agricultura como una actividad relevante para su economía, ya que proporciona alimentos a sus habitantes y aporta numerosos productos a las exportaciones. En México y en los países en vías de desarrollo, la agricultura tiene mayor peso, debido a que el 22,78\% de la población reside en el campo y aún desarrolla labores agrícolas, independientemente de que sea o no su principal fuente de ingresos (Bautista y Ramírez, 2008; Carton, 2009; Osorio-García et al., 2015), así como de seguridad alimentaria, ya que la mayoría de su producción es para autoconsumo (Guzmán et al., 2014).

De acuerdo con datos del censo general de población y vivienda 2010, el 22,2\% de la población mexicana vivía en zonas rurales, lo cual correspondió a 26 millones de habitantes aproximadamente (INEGI, 2016). En el año 2014, 55,3\% de la población mexicana vivía en algún grado de pobreza, ubicándose 38,3 millones en zonas urbanas y 17 millones en zonas rurales. Del total de la población que residía en zonas rurales, el 40,5\% se encontraba en pobreza moderada y el 20,6\% en pobreza extrema.

El maíz (Zea mays L.) y el frijol (Phaseolus vulgaris L.) son los dos cultivos más importantes en el sector agrícola mexicano (García et al., 2006), representan toda una tradición productiva y de consumo; cumplen diversas funciones alimentarias y socioeconómicas que le han permitido trascender hasta la actualidad (Ayala et al., 2008). Esta importancia deriva de su uso como ingredientes principales en la dieta de los mexicanos y como productos que aglutinan a más de dos terceras partes de los productores agrícolas del país. La producción de maíz y frijol, al igual que otros productos agrícolas del país, presenta el problema de falta de competitividad en el escenario actual de apertura comercial. Las causas y consecuencias de la pérdida de competitividad de la producción nacional son diversas (García et al., 2006), entre las cuales se pueden citar el poco acceso a programas federales y estatales de apoyo para el campo, la falta de créditos, las restricciones del minifundio, los factores climáticos y la disminución de la superficie cultivable, por el crecimiento de la mancha urbana estableciéndose en las mejores tierras de cultivo (Bobadilla et al., 2010). Otros factores de la pérdida de competitividad es el incremento en el precio de los insumos (principalmente fertilizantes), aunado a la disminución del precio de los granos en referencia.

Los programas de apoyo a los productores de maíz y frijol (PROMAF y PROCAMPO) son ineficientes en la asignación de los recursos al sector, dado que no llegan en tiempo y forma, es decir, se encuentran desfasados en su aplicación (González y Sánchez, 2008).

El maíz es uno de los principales granos cultivados en el mundo y su uso no sólo se centra en el consumo humano, sino que es un insumo importante en la producción del sector ganadero (Guzmán et al., 2014); desde el punto de vista alimentario, el maíz es el cultivo más importante de México. El promedio del consumo nacional aparente (CNA) entre 1980 y 1993 fue de 15,98 millones de toneladas (t); y de 1994 al 2013 este indicador se ubicó en 26,00 millones de toneladas. Sin embargo, considerando los últimos ocho años de la serie (2006-2013), el CNA fue de 30,28 millones de toneladas (FAOSTAT, 2016). El 87\% de los agricultores usan semilla de maíz criollo, adaptadas a las condiciones locales, pero de poca calidad para la industria (Herrera et al., 2002); el maíz blanco se utiliza en la industria de la harina como materia prima en la elaboración de tortillas, almidón y edulcorantes; mientras que en la elaboración de alimentos balanceados para el ganado se utiliza principalmente maíz amarillo (Retes, 2010). 
El frijol tiene gran importancia social, debido a que 570 mil personas se dedican y viven de este cultivo, con un promedio de cinco hectáreas por agricultor, los cuales destinan cerca del 20\% de la cosecha para autoconsumo; además, genera 78 millones de jornales al año; demanda en promedio 35 jornales por hectárea y en ello participa $71 \%$ de los integrantes de las familias involucradas, lo que constituye una forma muy importante de autoempleo (Serrano, 2004).

Este grano es básico en la dieta de la población mexicana, especialmente en la de medianos y bajos recursos, tanto en zonas rurales como urbanas, con un consumo anual per cápita de 12,5 kg (Ayala et al., 2008). Las tendencias de consumo per cápita a través del tiempo han ido en decremento, en la década de 1990 era de $13 \mathrm{~kg} / \mathrm{habitante} \mathrm{y}$ en la década del 2000 fue $11 \mathrm{~kg} /$ habitante (Rodríguez-Licea, 2010). Su importancia alimenticia radica en el aporte de grandes cantidades de proteína y fibra. El frijol es un alimento balanceado, pues es nutritivamente rico por su contenido de ácido fólico, fibra alimenticia y carbohidratos (Messina, 2014).

El consumo de frijol está dado principalmente por los factores económicos, culturales y naturales; en el primer caso, el consumo está dado por ingreso de las familias y en función del precio del grano (Rodríguez-Licea, 2010).

En el ámbito productivo el problema fundamental lo constituye la degradación, acidificación y erosión de los suelos, provocada principalmente por mal manejo, el minifundio con unidades de producción en promedio de 2,9 ha. Debido a la descapitalización del sector, la maquinaria con que se cuenta resulta insuficiente y en la mayoría de los casos se encuentra en mal estado, asimismo, se presenta baja productividad y elevados costos de producción, lo que origina baja competitividad en los mercados, además se registran escasos apoyos financieros para el sector agrícola (SEDAGRO, 2012).

En el 2010 México tenía una población de 118,6 millones de habitantes, de los cuales 26,1 millones (22\%) vivían en poblaciones rurales (FAOSTAT, 2016), en 2014 la población en pobreza eran 55,3 millones y en pobreza extrema 11,4 millones de habitantes, ubicados tanto en áreas urbanas como rurales (CONEVAL, 2015).

El maíz y el frijol en México son parte de la dieta de millones de personas, principalmente de la gente de bajos recursos económicos. La clasificación de las formas de producción y de propiedad en estos cultivos, es importante para la definición de los instrumentos de una política agrícola que se han implementado para impulsar la producción de maíz y frijol en México. Por lo anterior, el objetivo de este estudio fue evaluar la dinámica de la producción de maíz y frijol de México.

\section{Materiales y métodos}

Se analizaron los datos de producción de maíz y frijol en México entre los años de 1980 a 2014, considerando como variable al país en su conjunto.

La información estadística contenida en este estudio, pertenece a las publicaciones oficiales por parte de las distintas instituciones públicas. Los datos de superficie sembrada, producción, rendimientos y precios pagados al productor, se obtuvieron principalmente de la Secretaría de Agricultura, Ganadería, Desarrollo Rural, Pesca y Alimentación (SAGARPA) y sus organismos sectoriales; la información de las importaciones y exportaciones, fue obtenida de la Organización de las Naciones Unidas para la Alimentación y la Agricultura (FAO). Los datos de precios se deflactaron con el Índice Nacional de Precios al Productor (INPP), de junio del 2012, publicados por el Banco de México (Banxico) para pasarlos a pesos constantes. Adicionalmente, se tomaron estos precios y se realizó la conversión a dólares estadounidenses (USD); para ello se utilizó el tipo de cambio promedio del año 2012 (pesos por dólar) reportado por Banxico, el cual fue de 13,1689 pesos mexicanos por dólar. Se determinaron los incrementos/decrementos, la tasa de crecimiento media anual (TCMA), para la superficie sembrada, producción, rendimientos, precios, exportaciones e importaciones. Los datos se ajustaron por el método de los cuadrados mínimos para obtener la tendencia (Martínez, 1982), en todos los casos. 


\section{Resultados y discusión}

La superficie sembrada con maíz tuvo una tasa de crecimiento media anual de $-0,06 \%$, de 1980 a 2014; en 1980 se cultivaron 7597251 hectáreas (ha), cifra que en 2014 fue de 7426412 ha mostrando tendencia negativa; la disminución en el periodo de estudio fue de 170839 ha. La relación matemática obtenida fue y=8,4333-0,0153x, esta significa que por cada cambio unitario en la variable independiente (tiempo expresado en años), la superficie sembrada disminuye en 0,0153 millones de hectáreas, lo que es equivalente a 15300 ha por año. Dicha situación, se debe en buena medida al deterioro paulatino de los precios reales pagados a los productores por el grano, lo que los ha llevado a buscar alternativas de cultivos más rentables. Se observan variaciones en la superficie sembrada con maíz, en 1994 fue donde mayor superficie se sembró, con 9196478 ha (Figura 1). Por el contrario, de 1994 a 2014 hubo una disminución de 1,8 millones de hectáreas.

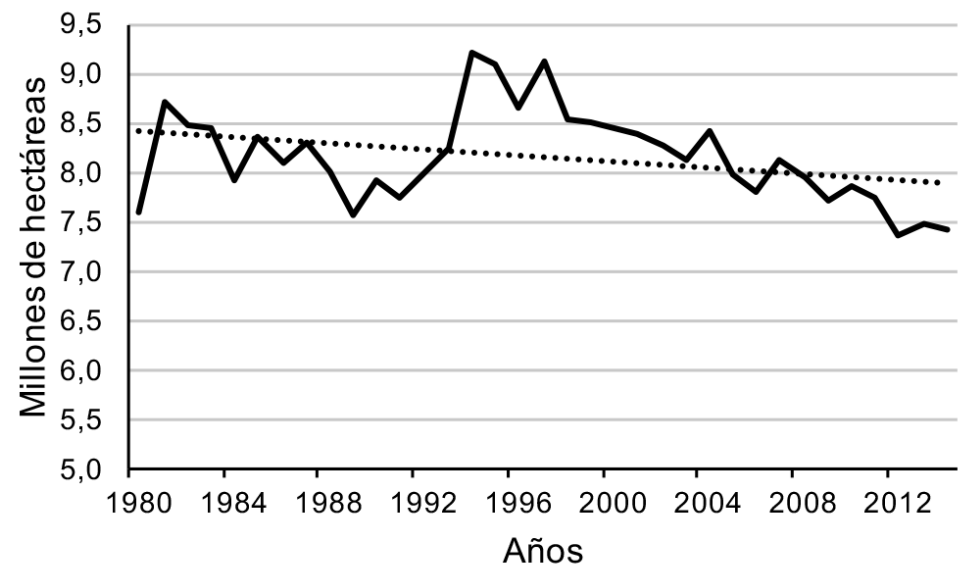

Figura 1. Superficie sembrada con maíz en México de 1980 a 2014.

Figure 1. Corn planted area in Mexico from 1980 to 2014.

La superficie sembrada con frijol mostró variación (Figura 2), con una tendencia negativa ( $\mathrm{y}=2,3694-0,0176 \mathrm{x}$ ), con una tasa de crecimiento media anual negativa de 0,24\%; pasó de 1967162 a 1773996 ha de 1980 a 2014, con una disminución de 193165 ha; en 1982 fue cuando mayor superficie cultivada hubo, con 2478869 ha, y el otro pico fue en 1999 con 2405873 ha; de 1999 a 2014 la disminución de la superficie sembrada fue 631876 ha. Al igual que en el caso del maíz, esta situación, se debe en buena medida al deterioro paulatino de los precios reales pagados a los productores por el grano, lo que los ha llevado a buscar alternativas de cultivos más rentables.

En ambos cultivos se registraron picos de producción en 1994, que fueron incentivados por las políticas agropecuarias implementadas para mitigar los posibles efectos negativos del Tratado de Libre Comercio de América del Norte (TLCAN), dirigidas a los productores de oleaginosas y granos básicos, a través del Programa de Subsidios Directos a la Agricultura (PROCAMPO). El frijol es definido en la Ley de Desarrollo Rural Sustentable de México (DOF, 2001) como un producto básico y estratégico para el desarrollo rural del país, ocupa el segundo lugar en superficie sembrada a nivel nacional, con un promedio de 1,85 millones de hectáreas. En los últimos años la superficie de maíz cosechada en temporal (6 millones de ha) y riego (1,1 millones de ha), han permanecido relativamente constantes. Resalta la drástica reducción en la superficie cosechada bajo temporal y el aumento de la superficie de riego en 2005, que hizo que la producción en ambos regímenes casi se igualara. La mayor parte 


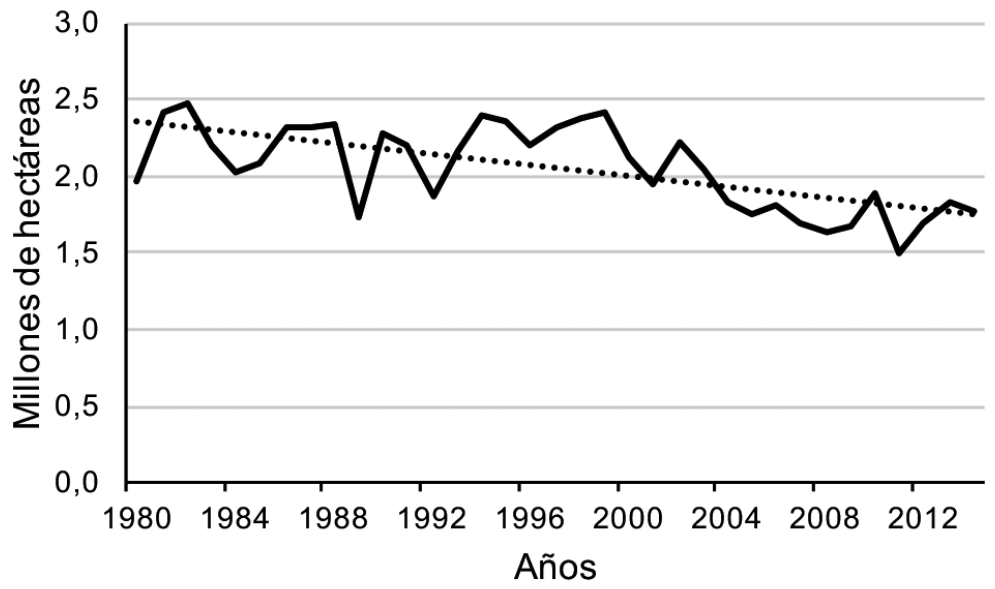

Figura 2. Superficie sembrada con frijol en México de 1980 a 2014.

Figure 2. Bean planted area in Mexico from 1980 to 2014.

del maíz bajo riego $(56,1 \%)$ se siembra en las entidades con zonas áridas (Márquez et al., 2006). Lo anterior, se puede explicar desde la perspectiva de los apoyos gubernamentales sobre el fomento a la comercialización con variedades que demanda el mercado nacional, así como paquetes tecnológicos adecuados, y sobre todo, productores comerciales de gran experiencia.

La disminución de la superficie sembrada con maíz y frijol significa que millones de productores dejan de cultivar sus tierras o buscan sembrar otros cultivos más rentables, con el consecuente deterioro de los ingresos rurales por los bajos precios pagados a los productores por sus cosechas (García et al., 2006) y la migración internacional principalmente a Estados Unidos de Norteamérica y la interna, que se da principalmente hacia las grandes ciudades de México (Carrera, 2008), engrosando las filas del desempleo o cinturones de miseria.

La producción de maíz en el periodo de 1980 a 2014 tuvo una tasa de crecimiento media anual de 1,82\%, con un incremento de 10,1 millones de toneladas, finalizando el 2014 con una producción de 23,3 millones de t y una tendencia de y=10,894+0,3656x. En la Figura 3, se muestra la producción de maíz, existiendo altibajos de 1985 a 1989, cuya disminución fue de 26,8\% equivalente a 3,2 millones de t; de 2008 a 2009 la disminución fue de 17,5\% con 4,3 millones de t, y de 2010 a 2011 la caída fue del 24,3\% y de 5,7 millones de toneladas. Este incremento se dio principalmente en utilización de semillas mejoradas y la utilización de fertilizantes.

En la producción de frijol existe una gran variabilidad como se muestra en la Figura 4, teniendo una tendencia positiva de $y=1063,3+2,3402 x$, con una tasa de crecimiento media anual de $0,89 \%$ y un incremento de $338783 \mathrm{t}$, en el 2014 la producción fue de 1273957 t; la producción promedio fue $1105454 \pm 235704$ t. En 1989 fue cuando se registró la menor producción con 593436 t y en 2002 la mayor producción, la cual ascendió a 1549091 t. Los incrementos se debieron principalmente a la utilización de semillas mejoradas en el sistema de producción de riego (FIRA, 2015).

Existe una gran variación en la producción de frijol, se dio una reducción del 50,1\% y 47,9\% con relación al año inmediato anterior, correspondiente a 2011 y 1992, respectivamente, los incrementos de 116,9 y 90,4\% corresponden a los años de 1990 y 2012 con su año inmediato anterior. En el 2014 la superficie sembrada de frijol en temporal fue del 86,6\% (1 535826 ha) y aportó el 72,6\% de la producción (924 892 t). En los últimos años, el $70 \%$ de la producción de frijol se obtiene de superficies de temporal (Ortega y Ochoa, 2003), la cual se ve 


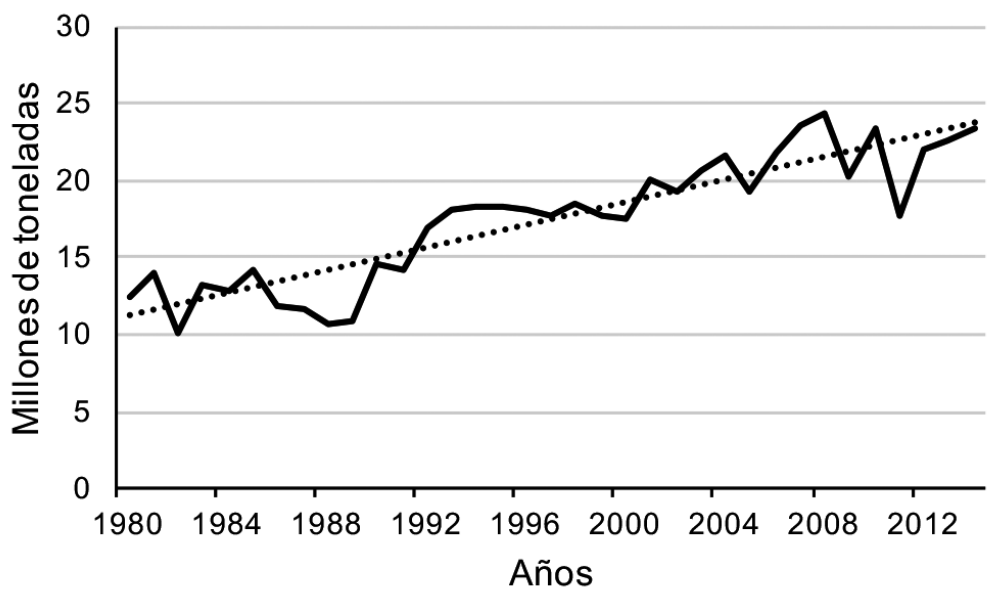

Figura 3. Producción de maíz en México de 1980 a 2014.

Figure 3. Corn production in Mexico from 1980 to 2014.

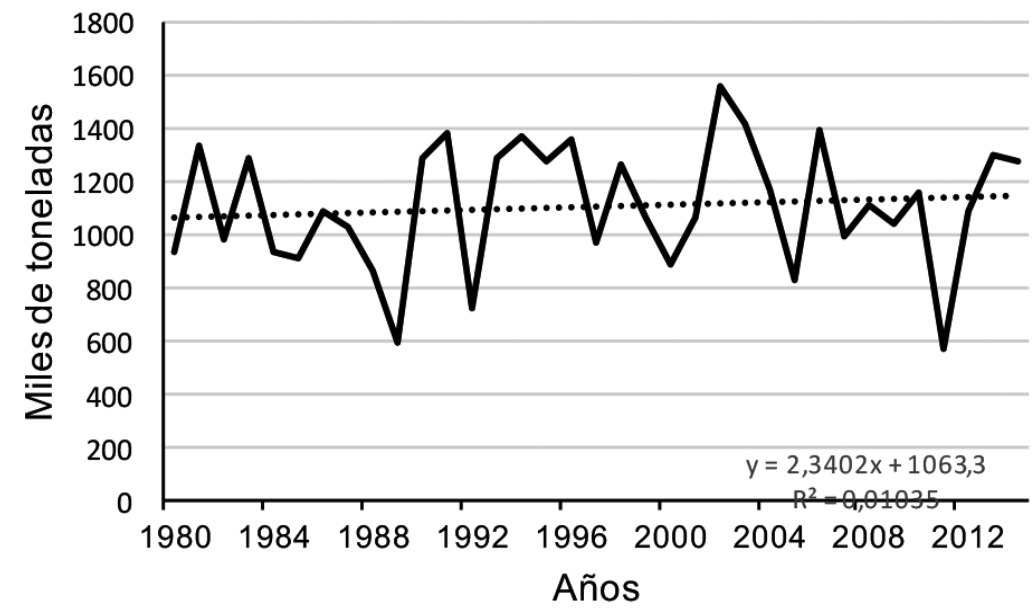

Figura 4. Producción de frijol en México de 1980 a 2014.

Figure 4. Bean production in Mexico from 1980 to 2014.

seriamente afectada en los rendimientos por factores como sequías, exceso de lluvias, heladas tempranas y al ataque de plagas y patógenos (Márquez et al., 2006).

La tasa de crecimiento media anual del rendimiento del maíz por hectárea en el período de 1980 a 2014 fue 1,7\%, pasó de 1,83 a 3,30 t/ha con incremento de 1,47 t durante 35 años, la cual tiene una tendencia de $\mathrm{y}=1,5067+0,0519 x$ (Figura 5).

El rendimiento por hectárea de maíz en el periodo de estudio presentó una TCMA de 1,7\%, sin tomar en cuenta el régimen de producción (temporal o riego); la productividad del maíz sembrado en temporal ha permanecido constante, ya que entre 1980 y 2005 aumentó en solo un 19\%; caso opuesto ocurre en las zonas de riego, donde el incremento en los rendimientos entre 1980 a 2005 fue del 144\%, lo que equivale a una tasa de crecimiento anual del 3,5\%, pasó de 2,73 a 6,61 t/ha, debido a los avances logrados en los estados de Sinaloa y Chihuahua (García et al., 


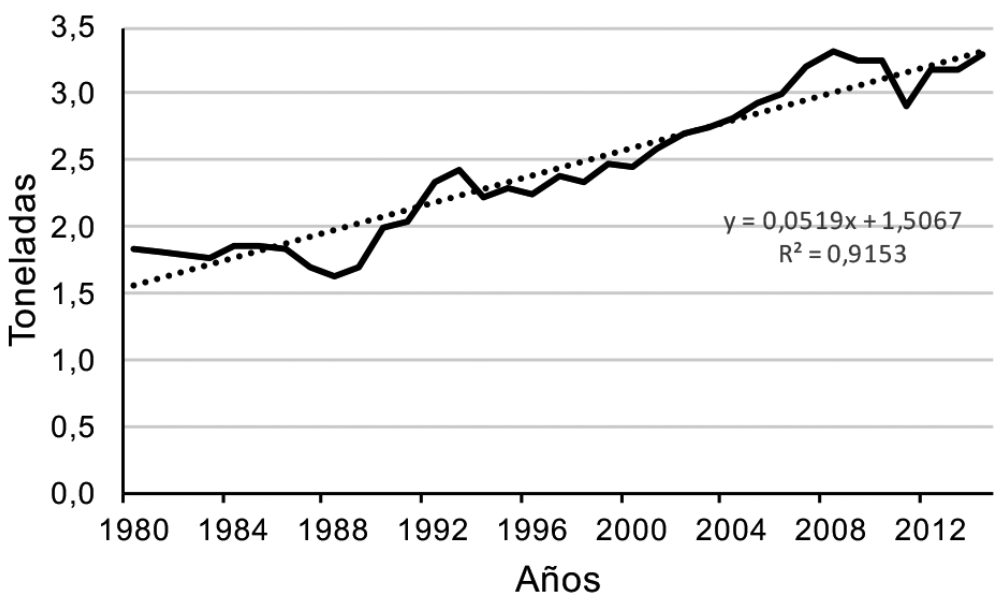

Figura 5. Rendimiento de maíz en toneladas/hectárea en México de 1980 a 2014.

Figure 5. Tons/hectare corn yield in Mexico, from 1980 to 2014.

2006). Moreno (2014) reporta que la producción bajo riego se ha incrementado de 1980 a 2012 con una tasa de crecimiento media anual de $3,46 \%$, mientras que la producción de temporal tiene una TCMA de $0,94 \%$. En cuanto a la producción de maíz en temporal, se ha pasado de producir 9,3 millones de toneladas en 1980 a un máximo de 14 millones de toneladas en 2003, reduciéndose a 10,3 millones de toneladas en 2005 por las condiciones desfavorables climatológicas (Márquez et al., 2006).

Se estima que los productores de temporal de maíz operan al 57\% de su potencial productivo, con un déficit de rendimiento de $43 \%$ (FAO, 2011). La mayor parte de las regiones operaban a menos de $50 \%$ de su potencial (maquinaria obsoleta, mal uso de fertilizantes y poco uso de semillas mejoradas), eliminar este déficit de rendimiento añadiría más de nueve millones de toneladas a la producción nacional (Turrent, 2009; Turrent et al., 2012).

De acuerdo con Flores et al. (2007), el 43\% de la producción nacional de maíz es consumida por el sector pecuario; un $34 \%$ es usada para la producción de tortillas, $21 \%$ es demandada por el sector industrial, $2 \%$ se usa como simiente y tan solo el $18,4 \%$ del volumen total producido se destina al autoconsumo $(11,1 \%$ corresponde al consumo directo en forma de tortilla y en comunidades rurales y el otro 6,9\% se destina al consumo del ganado de solar).

Los rendimientos del frijol por hectárea pasaron de 600 a $760 \mathrm{~kg}$, con una tasa de crecimiento media anual de $0,68 \%$ durante 35 años que comprende el periodo de estudio, con altibajos en dicho rubro; en 1988 se registró el menor rendimiento con $440 \mathrm{~kg} / \mathrm{ha}$ y el más alto en 2009 con $860 \mathrm{~kg} / \mathrm{ha}$ (Figura 6). El incremento que tuvo en el periodo de estudio fue de $160 \mathrm{~kg} / \mathrm{ha}$.

El referido incremento en rendimientos se dio principalmente por la introducción de mejores paquetes tecnológicos, incentivados por algunos programas gubernamentales, como es el caso del Programa de maíz, arroz y frijol (PROMAF), el cual entrega recursos económicos a los productores, servicios de asistencia técnica, capacitación, innovación tecnológica, desarrollo organizativo, así como la inducción hacia la agricultura sustentable y el uso de crédito para capitalizarse y mejorar su rentabilidad.

Los bajos rendimientos son mayores entre los pequeños y medianos productores de maíz y frijol de temporal, esta observación es consistente con evaluaciones internacionales (FAO, 2011). Como este sector de productores tiene acceso limitado al crédito formal, al riego y ha sido desatendido por los servicios de extensión en las últimas dos décadas, los productores producen por debajo de su potencial, el sector también sufre de organización inoperante que limita cualquier incremento en la productividad (Turrent et al., 2012). El porcentaje de productores 


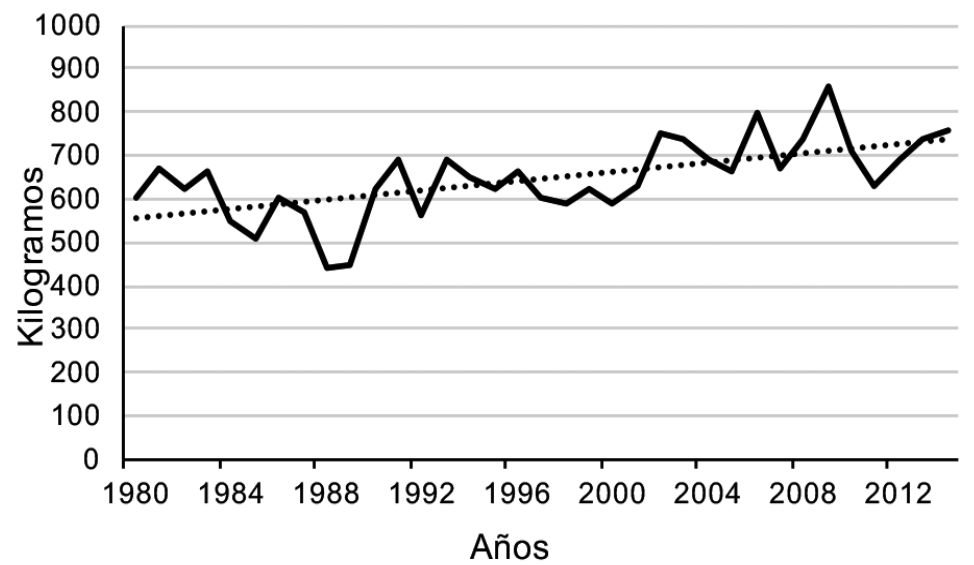

Figura 6. Rendimiento de frijol en kg/ha en México de 1980 a 2014.

Figure 6. $\mathrm{Kg} / \mathrm{ha}$ bean yield in Mexico, from 1980 to 2014.

que tiene acceso a créditos y seguros para el sector fue del 11,5\%, alrededor 364543 agricultores de maíz y frijol divididos en dos grupos, campesinos medianos con una superficie promedio de 4,3 ha y los empresario campesinos con una superficie promedio de 13,3 ha (González-Estrada, 2009)

El frijol producido en México no se destina en la mayor parte al autoconsumo, se trata de una actividad agrícola predominantemente integrada al mercado y a la producción mercantil (González-Estrada, 2009); FIRA (2001) reportó que el $20 \%$ de la producción nacional de frijol se destina al autoconsumo y un 5\% se usa como simiente; los mayoristas de las centrales de abasto compran el 39\%, las empacadoras el 26\%, se industrializa el $5 \%$ y las pérdidas por transporte, plagas y mermas en general, fue de 5\%. En 2009 la producción fue 1207000 t, de las cuales el 94\% fue comercializable y el resto fue para autoconsumo (SAGARPA, 2011). Para el 2014 el INEGI (2016), reportó en la Encuesta Nacional Agropecuaria, que el 20,3\% de la producción de frijol es para autoconsumo, el 62,7\% se vende a intermediarios, $2,3 \%$ se coloca directamente al consumidor y el 7,4\% se comercializa a empacadoras o industria procesadoras; estos datos concuerdan con lo reportado por FIRA (2001) respecto a la producción destinada al autoconsumo, y el resto se comercializa de diferentes formas.

El precio del maíz a precios constantes deflactados con el INPP de junio de 2012, y transformados de pesos mexicanos a dólares estadunidenses, tuvo una disminución de 441,9 dólares/tonelada durante el periodo de estudio; en 1981 se pagó 684,5 USD/t y en 2014 finalizó en 242,6 USD/t, la disminución fue del 64,4\% en 34 años. Durante 1982, 1984-1985, 1987, 1995, 2000, 2003, 2006-2008 y 2010-2011, el precio del maíz se incrementó en 3,4, 13,4, $18,5,37,2,5,8,2,4,45,3$ y $36,1 \%$ con respecto a su año inmediato anterior, equivalente a $23,6,89,4,124,2,111,1$, 14,8, 5,5, 96,7 y 91,1 dólares por tonelada, respectivamente. Por otro lado, los años de 1983, 1986, 1988-1994, 1996-1999, 2001-2002, 2004-2005, 2009 y 2012-2014 los precios disminuyeron en 6,8, 10,3, 88,8, 44,6, 14,5, 19,9, 14,4 y $30,6 \%$, con respecto a su año inmediato anterior, equivalentes a $-48,1,-77,1,-217,8,-154,6,-38,1,-46,1$, $-41,4$ y $-94,7$ dólares por tonelada, respectivamente (Figura 7).

El precio pagado del frijol a precios constantes tuvo una disminución de 976,3 USD/t, equivalente al 59,0\%, durante los 34 años del periodo de estudio, en 1981 el precio fue de 1654,4 USD/t. Durante 1984-1985, 1990, 19961997, 2000-2001, 2004-2005, 2007-2009 y 2011 los precios del frijol se incrementaron en 102,1, 46,5, 51,9, 12,6, $12,0,36,7$ y $27,1 \%$ con respecto a su año inmediato anterior, equivalente a 1127,3, 491,2, 472,8, 117,7, 91,6, 308,8 y 214,2 dólares por tonelada, respectivamente. Por otro lado, en los años de 1982-1983, 1986-1989, 1991-1995, 19981999, 2003-2004, 2006, 2010 y 2012-2014 los precios disminuyeron en 35,0, 65,7, 58,8, 30,4, 30,7, 10,3, 25,4 y 


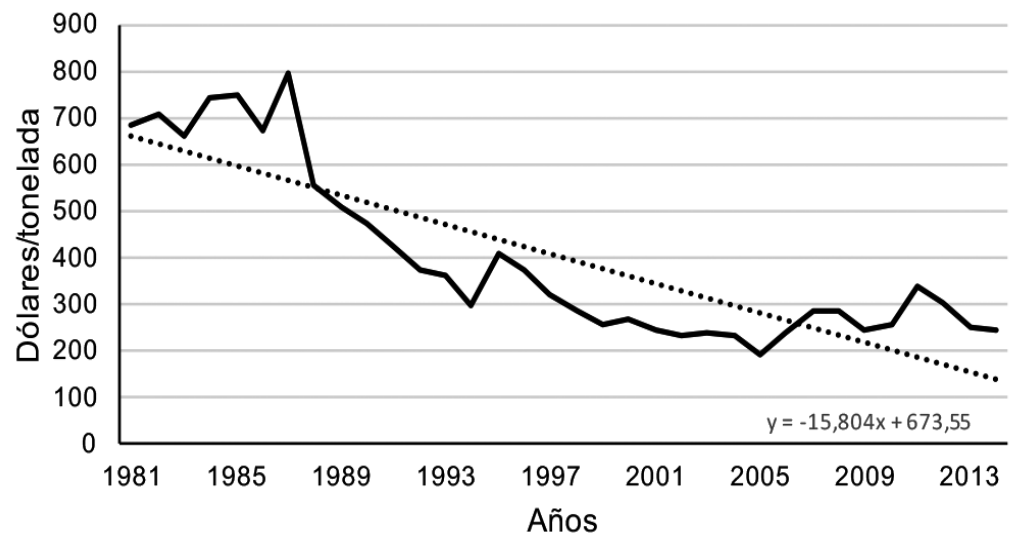

Figura 7. Precios constantes del maíz (dólares/t) en México de 1980 a 2014.

Figure 7. Corn constant prices (dollars/t) in Mexico, from 1980 to 2014.

$35,4 \%$ con respecto a su año inmmediato anterior, equivalente a $-552,6,-1173,3,-726,3,-372,4,-294,5,-86,4,-268,3$ y $-325,6$ dólares por tonelada, respectivamente (Figura 8).

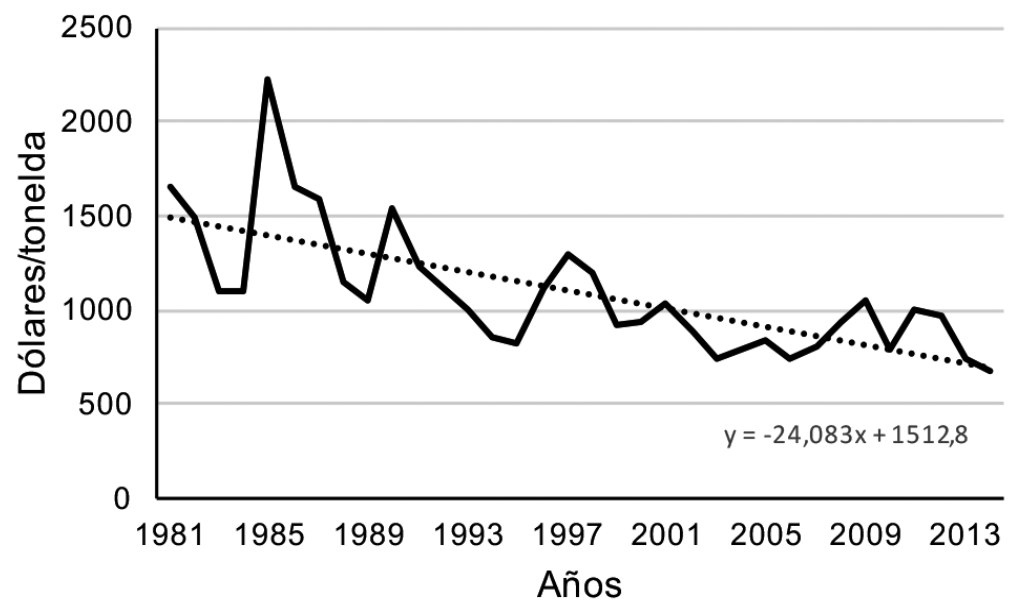

Figura 8. Precios constantes del frijol (dólares/t) en México de 1980 a 2014.

Figure 8. Bean constant prices (dólares/t) in Mexico, from 1980 to 2014.

Los precios pagados a los productores de maíz y frijol tuvieron una tendencia negativa; el precio pagado de maíz por tonelada implicó que el productor que lleva en la misma actividad durante 34 años, dejó de percibir un acumulado de 441,9 USD/t; para el caso del frijol el productor dejó de percibir 976,3 USD/t durante el periodo de estudio.

El deterioro de los precios se debió básicamente a que los precios de los productos en referencia crecieron menos que la inflación, es decir, disminuyeron en términos reales. De acuerdo con información reportada por el INEGI (2016), el índice inflacionario para enero de 1980 fue de 0,07, mientras que en diciembre de 2014 fue de 
116,06; otro factor que influyó en la disminución de los precios fue la depreciación del peso con respecto al dólar, por tanto, al efectuar la conversión, esta tiende a ser menor. En enero de 1980, el dólar se cotizó en 0,023 pesos, y en diciembre de 2014 el tipo de cambio fue de 14,74 pesos por dólar (Banxico). Lo anterior, trae como consecuencia que deje de haber incentivos para que los productores sigan cultivando tales productos y se desplacen hacia otros cultivos, o incluso, a otras actividades.

De 1988 a 1999 la disminución de los ingresos por los precios pagados a los productores de maíz fue de 96,2\%, equivalente a 541,7 USD/t, aunque en 1995 hubo un incremento de 37,2\% correspondiente a 111,7 USD/t, no compensó lo perdido de 1988 a 1999; en 1988 el pago por tonelada fue 556,4 dólares. Los periodos donde el frijol tuvo disminuciones pronunciadas fueron de 1986-1989, 1991-1995 y 1982-1983 los más fuertes con 1173,3; 726,3 y 552,6 USD/t, respectivamente, y un acumulado de 2452,2 USD/t; por otro lado, los periodos con mayor incremento fueron 1984-1985, 1990 y 1996-1997 de 1127,3 491,2 y 472,4 USD/t. Los incrementos no compensan lo perdido, se mantuvo un saldo negativo de 360,9 dólares por tonelada. En el periodo 2000-2002 los precios reales del grano de maíz disminuyeron en 36,9 \% respecto al periodo 1991-1993 (Calva et al., 2006). Entre 1990 y 2005 los precios reales del frijol cayeron a una tasa promedio anual de 4\%, y aun con el subsidio de PROCAMPO, los precios de este cultivo presentan un decrecimiento a una tasa anual de 2,9\%, existió una pérdida en el poder adquisitivo de los productores de frijol con una tendencia decreciente (Márquez et al., 2006). Haciendo el análisis de 1995 a 2014, se tuvo una reducción de 166,83 USD/t en el precio pagado al productor de maíz, y en caso del frijol fue de 141,93 USD/t. Lo anterior representa que el precio del maíz cayó a una tasa promedio anual de 2,58\% y el del frijol en $0,95 \%$, en el referido periodo, aun con el apoyo de PROCAMPO. La disminución en el ingreso de los productores originó la descapitalización del campo, el aumento en la pobreza rural, el desempleo y la migración (Dussel, 2004).

En los últimos años, los productores mexicanos de frijol han enfrentado diversos problemas que han repercutido en una reducción de su rentabilidad y competitividad, lo cual puede demostrarse haciendo un análisis de la evolución de los precios de los insumos, según se detalla líneas abajo; en relación con el principal socio comercial mexicano, Estados Unidos, el rendimiento nacional promedio de 2003 a 2005 fue de 0,65 toneladas por hectárea (SAGARPA-SIACON, 2006), que representó menos de la mitad del obtenido por los estadounidenses, de $1,8 \mathrm{t} / \mathrm{ha}$ en promedio, en el mismo lapso (USDA-ERS, 2006).

Aunado a lo anterior, los costos en México son altos, y los agricultores enfrentan un deterioro continuo de los precios reales del grano. Entre 1980 y 2005, el índice de precios de insumos creció a una tasa promedio anual de 30,4\%, mientras que el de precios al productor decreció en 2,1\% (Banco de México, 2005), y los rendimientos apenas aumentaron 0,4\% (SAGARPA-SIACON, 2006), lo que implica una reducción en la rentabilidad. En el periodo 2000-2014 hubo un incremento en el precio de los fertilizantes, ejemplo de ello, es que la urea pasó de 152,78 USD/t a 358,49 USD/t, teniendo un incremento de 134,65\%; el 18-46 (DAP) se incrementó 228,38 USD/t, en el año 2000 costó 211,46 USD/t, y en el 2014, 439,84 USD/t; finalmente, el cloruro de potasio tuvo un incremento de $166,46 \%$, pasando de 163,26 a $439,84 \mathrm{USD} / \mathrm{t}$.

Las importaciones de maíz tuvieron una TCMA de 2,84\%, en 2012 con un acumulado de 9,5 millones de toneladas, mostró un incremento de 5,7 millones de toneladas con respecto a 1980 (Figura 9). En el periodo de 1980 a 2012, existió gran variación de las importaciones de este grano en 1993 fue cuando menor cantidad se introdujo al país (210 mil t); los años con mayor variación fueron 1994, 1983, 1987 y 1998, con un incremento de 1203,9, 1166,0, 111,5 y $106,9 \%$, equivalente a 2,5, 4,3, 1,9 y 2,7 millones de toneladas con respecto a su año inmediato anterior.

Las exportaciones de maíz no sobrepasaron el millón, con una gran variación entre los distintos años, la cual no mostró una tendencia; en 2012 fueron de 773 mil t y en 1980 de 90 t, los mayores incrementos se dieron en 1983, $1991,2002,1981$ y 2005 con 8520,7, 2029,6, 1363,8, 782,8 y 651,6\% equivalente $20620,15425,153197,728$ y 45983 toneladas respecto a su año inmediato anterior, respectivamente; los decrementos fueron en 1999, 2003, 


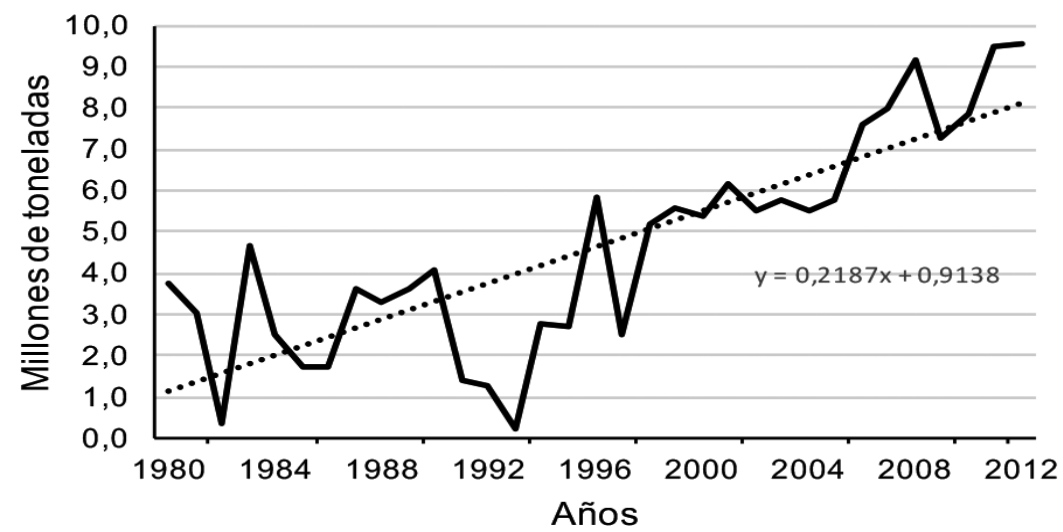

Figura 9. Importaciones de maíz de México de 1980 a 2014.

Figure 9. Mexico corn imports, from 1980 to 2014.

2011, 1984 y 1989 con 92,3, 91,2, 81,9, 76,5 y 76,1\% equivalente a $-213455,-150$ 017, - 457 598, - 15953 y -5050 toneladas respecto a su año inmediato anterior, respectivamente.

Las importaciones de frijol (Figura 10) han tenido una gran variabilidad durante los años de 1980 a 2012, con base en los datos reportados por la FAO; en 1982 fue cuando se introdujo al país la mayor cantidad de frijol con 482 mil toneladas, y el año con menores compras al exterior fue 1992 con 2909 toneladas. Las variaciones de 659,1, 401,8, $199,4,165,2$ y $160,2 \%$ correspondientes con un incremento de 49 939, 104 718, 220101,68758 y 4662 toneladas con respecto a su año inmediato anterior de 1994, 1996, 1990, 1988 y 1996, respectivamente; mientras que los mayores decrementos se dieron en 1990-1991, 1982-1984, 1987 y 1995 con 181,2, 108,3, 77,9 y 54,7\%, correspondiente a -327 $562,-363001,-139481$ y -31 348 toneladas con respecto a su año inmediato anterior, respectivamente.

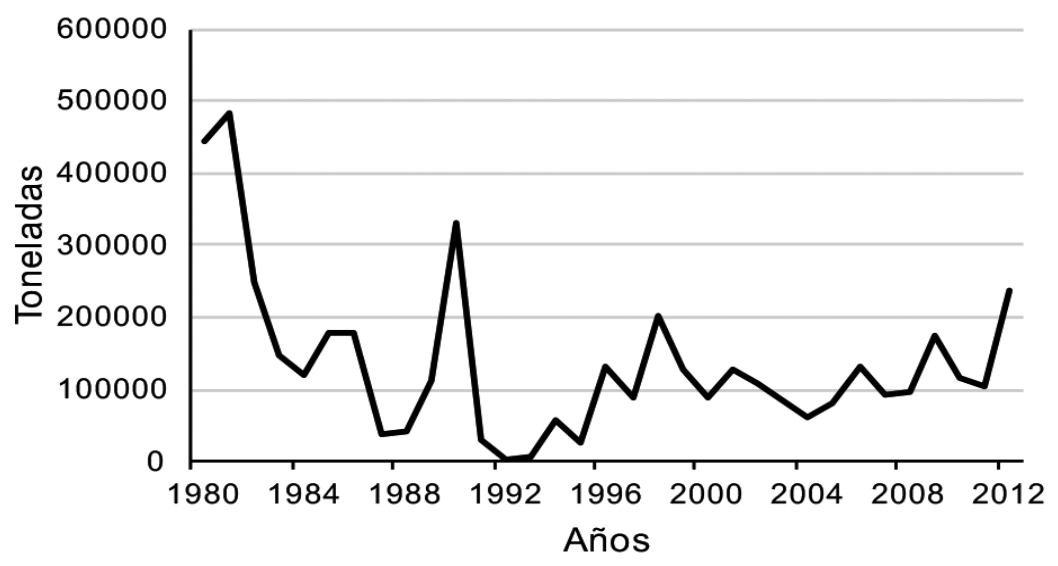

Figura 10. Importaciones de frijol de México de 1980 a 2014.

Figure 10. Mexico bean imports, 1980 to 2014.

En cuanto a las exportaciones, han tenido un comportamiento irregular; en 1986 no hubo ventas al exterior, y en 1984 el volumen del comercio fue 130 mil toneladas, con altibajos, en 1998 tuvo un incrementos del 12 509\% (pasó de 69 a 8696 t) y disminuciones del $86,45 \%$ con respecto a su año inmediato posterior. 
Las importaciones de maíz en 1994 aportó al consumo nacional el 13,1\% y en el 2012 el 30,9\%, teniendo una tendencia creciente, siendo cada vez más dependientes de las importaciones para abastecer el consumo del pueblo mexicano (Figura 11); para el caso del frijol, el porcentaje de participación de las importaciones en el consumo nacional fue variable del $2 \%$ hasta el $18,1 \%$ en el 2012 , manteniendo una tendencia positiva a incrementar las importaciones para satisfacer la demanda interna (Figura 11).

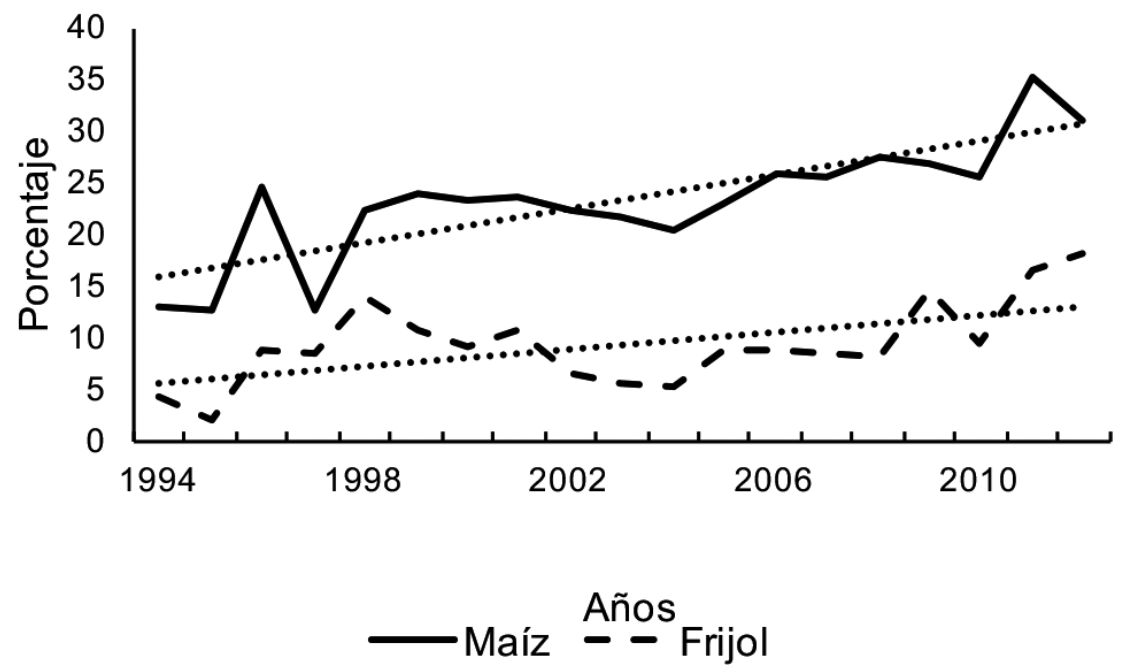

Figura 11. Porcentaje de las importaciones de maíz y frijol en el consumo de México de 1994 a 2014.

Figure 11. Percentage of consumption of corn and beans imports in Mexico, from 1994 to 2014.

México fue el país con los precios más altos al momento de la entrada en vigor del Tratado Libre Comercio con América del Norte (TLCAN) (San-Juan-Mejía et al., 2007). Los costos promedios de producción de frijol durante el periodo de 1989 a 1991, comparados entre México y Estados Unidos, fueron México 641,17 y Estados Unidos 219,53 dólares por tonelada, y los precios pagados al productor para México fue de 631,42 y Estados Unidos de 460,00 dólares por tonelada (Ayala et al., 2008). El descenso de precios puede tener consecuencias en el mercado doméstico, como por ejemplo, un menor ingreso de los productores, menor participación de mercado, menor empleo de insumos (mano de obra entre ellos) y un descenso en la capacidad instalada (San-Juan-Mejía et al., 2007).

Del análisis sobre la producción y consumo de maíz en México se rescata que a pesar de que la producción del grano ha aumentado a nivel nacional, no lo ha hecho al mismo ritmo que el consumo, por tanto, la brecha entre estos es cada vez más amplia (Moreno, 2014), por lo cual se ha recurrido a las importaciones para satisfacer el mercado nacional. La producción nacional de maíz blanco cubre en su totalidad la demanda; para el caso del maíz amarillo, que se utiliza en el sector pecuario en su mayor parte, es importado (Retes, 2010). Del total de las importaciones, el 93\% de las compras corresponden al maíz amarrillo destinado a la industria pecuaria y almidonaria principalmente (González y Ávila, 2014).

Desde el punto de vista del comercio exterior, el grano de maíz es el rubro más importante de las importaciones de productos agropecuarios del país (González y Alferes, 2010). En cuanto al índice de dependencia a las importaciones de maíz, se ha incrementado fuertemente, en especial después de la firma del TLCAN, a pesar del incremento en la producción nacional originado en el mismo periodo. Al estimar el índice de dependencia sobre 
el consumo de maíz en México, se observa que en el año 2011, el 35\% del maíz que se utilizó en México fue importado (Moreno, 2014), principalmente de Estados Unidos.

Las importaciones mexicanas de maíz tuvieron un incremento en el periodo de estudio de 39,7\%, el incremento fue de 5,7 millones de toneladas, finalizó en el 2013 con 7,2 millones de toneladas; existiendo dos periodos críticos de 1982 a 1990 y de 1993 a 2012, donde el incremento fue de 586447 t y 9,4 millones de toneladas con una TCMA $30,6 \%$ y $21,0 \%$, respectivamente; el último periodo, coincidente con la entrada de vigor del TLCAN. Con respecto a las importaciones de frijol, en 1982 fue donde se importaron el mayor número de toneladas con 482 mil y la menor cantidad en 1992 con 2909 t. En el periodo de 1992 a 2013, que coincidió con la entrada en vigor del TLCAN, la TCMA fue de 19,0\%, el incremento fue de 131,6 mil t, con altibajos en el periodo, en los años de 2012 y 1998 de mayor introducción de esta leguminosa con 236 mil y 202 mil t. El cultivo de maíz es, sin duda, el más importante del país y resume en buena medida la naturaleza y los problemas de la agricultura mexicana (González-Estrada, 2009).

La dependencia alimentaria de México, en caso de los dos cultivos en estudio, ha ido en incremento, sobre todo a partir de la entrada en vigor del TLCAN, en 1994 la dependencia en maíz fue de 13,1\% y en 2013 pasó al 24\%; en frijol, para 1994 dicho indicador fue de 4,4\% y en 2013 pasó a 9,6\%, aunque cabe hacer mención que en el año 2012 fue de $18,1 \%$. Si el consumo de maíz y frijol dependiera en gran parte de las importaciones, el país estará expuesto a las fluctuaciones de los precios internacionales, la escasez de alimentos por factores climáticos y la demanda de otros países, es otro argumento a favor de la protección de estos dos cultivos básicos para el pueblo mexicano. Mediante la protección, el Gobierno Federal debería estimular la producción a tal grado de depender lo menos posible de las importaciones (García et al., 2006). La protección de las importaciones no es la única medida que debe adoptar el gobierno, sino a través de mejorar la eficiencia en la producción, comercialización y transformación, para que los productores tengan mejores beneficios y mejor calidad de vida. El cambio climático afectará la producción y los ingresos agrícolas en México; en algunas partes del país, la producción de maíz y frijol decrecerá por el incremento en la temperatura y reducción de las precipitaciones, así como por la mayor recurrencia de eventos extremos como sequías e inundaciones (SAGARPA, 2011).

La pérdida de autosuficiencia alimentaria de los productos más importantes en la dieta de los mexicanos, ocasiona dependencia y vulnerabilidad hacia las fluctuaciones de los precios internacionales. Las importaciones agroalimentarias aumentaron de 2755,7 millones de dólares por año en el trienio 1980-1982, a 5582,1 millones de dólares en 1991-1993, y a 10 870,8 millones de dólares en 2000-2002 (Calva et al., 2006).

El cambio de la política económica desarrollada durante los últimos 35 años no ha incentivado la producción de maíz y frijol en México; por el contrario, ha estimulado las importaciones, acentuándose con la entrada en vigor del Tratado de Libre Comercio de América del Norte.

Debería implementarse una política de estado que impulse la producción de estos dos granos básicos en la dieta de los mexicanos, de tal forma que repercuta en un incremento en la rentabilidad de los productores de dichos cultivos, con el consecuente beneficio para los productores y se disminuya la dependencia a las importaciones, salvando con ello la soberanía alimentaria del país.

\section{Literatura citada}

Ayala, A.V., R. Schwentesius, G. Almaguer, y A. Gómez. 2008. Competitividad del frijol mexicano frente al de Estados Unidos en un contexto de liberalización comercial. Región y Sociedad 20(42):37-72.

Bautista, J.A., y J. Ramírez. 2008. Agricultura y pluriactividad de los pequeños productores de agave en la región del mezcal, Oaxaca, México. Agric. Téc. Méx. 34:443-451. 
Banco de México. 2005. Índices de precios. http://www.banxico.org.mx/SieInternet/consultarDirectorioInternetAction.do?acci on $=$ consultarCuadro\&idCuadro $=\mathrm{CP} 171 \&$ locale $=$ es (consultado set. 2015).

Bobadilla, S.E., G. Rivera, y L. Del Moral. 2010. Factores de competitividad del cultivo de lechuga en Santa María Jajalpa, Estado de México. Análisis Económico 59:143-154.

Calva, J.L., R. Schwentesius, y A. Gómez. 2006. La economía mexicana en el décimo año del TLCAN y reflexiones sobre la agricultura. http://fdclberlin.de/fileadmin/fdcl/rita052004.pdf (consultado oct. 2015).

Carton, G.H. 2009. La nueva estructura ocupacional en los hogares rurales mexicanos En: G.H. Carton, y V.L. Martínez, editores, La pluriactividad en el campo latinoamericano. FLACSO, ECU. p. 273-307.

Carrera, C.B. 2008. La ovinocultura en México: alternativa para los productores rurales? Avances Cuadernos de Trabajo 207:1-17.

CONEVAL (Consejo Nacional de Evaluación de la Política de Desarrollo Social). 2015. Medición de la pobreza en México y en las entidades federativas 2014. http://www.coneval.org.mx/Medicion/Documents/Pobreza\%202014_CONEVAL_web. pdf (consultado jun. 2016).

DOF (Diario Oficial de la Federación). 2001. Ley de desarrollo rural sustentable. http://www.diputados.gob.mx/LeyesBiblio/ pdf/235.pdf (consultado oct. 2015).

Dussel, P.E. 2004. Efectos de la apertura comercial en el empleo y el mercado laboral de México y sus diferencias con Argentina y Brasil (1990-2003). Unidad de Análisis e Investigación sobre el Empleo, Ginebra, SUI.

FAO. 2011. The state of the world's land and water resources for food and agriculture. Managing systems at risk. FAO, Rome, ITA.

FAOSTAT. 2016. Composición de la población en México. http://faostat3.fao.org/browse/area/138/S (consultado jun. 2016).

FIRA (Fideicomisos Instituidos en Relación a la Agricultura). 2015. Panorama Agroalimentario Frijol 2015. https://www.gob. mx/cms/uploads/attachment/file/61950/Panorama_Agroalimentario_Frijol_2015.pdf (consultado jun. 2016).

FIRA (Fideicomisos Instituidos en Relación a la Agricultura). 2001. El frijol en México Competitividad y oportunidades de desarrollo. Boletín Informativo No. 316. FIRA, Morelia, MEX.

Flores, V.C., P. Ponce, y P. Ramírez. 2007. Situación del maíz y la tortilla. CIESTAAM, Universidad Autónoma Chapingo, Estado de México, MEX.

García, J.A., G. Rodríguez, A. Sáenz, y S. Rebollar. 2006. Políticas para mejorar la competitividad de la producción de maíz y frijol en México. Rev. Fitotec. Mex. 29:115-121.

González, E.A., y M. Alferes. 2010. Competitividad y ventajas competitivas de la producción de maíz en México. Rev. Mex. Cienc. Agríc. 1:381-396.

González, M.A., y J. Ávila. 2014. El maíz en Estados Unidos y en México. Hegemonía en la producción de un cultivo. Argumentos 75:215-237.

González, E.A., y Y. Sánchez 2008. Ineficiencia de las transferencias del estado a la agricultura mexicana. Rev. Mex. Ec. Agríc. y de los Rec. Nat 1:7-26.

González-Estrada, A. 2009. Estimación de las estructuras agrarias y económicas de la producción de maíz y frijol en México. Rev. Mex. Ec. Agríc. y de los Rec. Nat. 2(1):7-30.

Guzmán, S.E., M. De-la-Garza, J. González, y J. Hernández. 2014. Análisis de los costos de producción de maíz en la Región Bajío de Guanajuato. Análisis Económico 70:145-156.

Herrera, B.E., A. Macías, R. Díaz, M. Valadez, y A. Delgado. 2002. Uso de semilla criolla y caracteres de mazorca para la selección de semilla de maíz en México. Rev. Fitotec. Mex. 25:17-23. 
INEGI (Instituto Nacional de Estadística y Geografía). 2016. Encuesta nacional agropecuaria. 2014. http://www.inegi.org.mx/ saladeprensa/boletines/2015/especiales/especiales2015_08_8.pdf (consultado set. 2016).

Martínez, G.A. 1982. Métodos econométricos. Colegio de Posgraduados, Motecillos, Estado de México, MEX.

Márquez, B.S., R. Schwentesius, A. Ayala, y A. Kalil 2006. La globalización y su efecto en la producción agrícola de las zonas áridas y semiáridas de México. Rev. Chapingo Serie Zonas Áridas 5:107-116.

Messina, V. 2014. Nutritional and health benefits of dried beans. Am. J. Clin. Nutr.100:437-44.

Moreno, L.I. 2014. Dependencia de México a las importaciones de maíz en la era del TLCAN. Tesis MSc., Colegio de la Frontera Norte, Baja California, MEX.

Osorio-García, N., H. López-Sánchez, B. Ramírez-Valverde, A. Gil-Muñoz, y N. Gutiérrez-Rangel. 2015. Producción de maíz y pluriactividad de los campesinos en el Valle de Puebla, México. Nova Scientia 14:577-600.

Ortega, R.C., y R. Ochoa 2003. El frijol mexicano y el nuevo siglo. Claridades Agropecu. 124:6-15.

Retes, R.F. 2010. Demanda de tortilla de maíz en México, 1996-2008. Tesis Dr., Colegio de Posgraduados, Texcoco, Estado de México, MÉX.

Rodríguez-Licea, G., J. García-Salazar, S. Rebollar-Rebollar, y A. Cruz-Contreras. 2010. Preferencias del consumo de frijol (Phaseolus vulgaris L.) en México: factores y características que influyen en la decisión de compra diferenciada por tipo y variedad. Paradigma Económico 2(1):121-145.

SAGARPA (Secretaría de Agricultura, Ganadería, Desarrollo Rural, Pesca y Alimentación). 2011. Estudio de gran visión y factibilidad económica y financiera para el desarrollo de infraestructura de almacenamiento y distribución de granos y oleaginosas para el mediano y largo plazo a nivel nacional. MEX. http://www.sagarpa.gob.mx/agronegocios/documents/ estudios_promercado/granos.pdf (consultado jun. 2015).

SAGARPA-SIACON (Secretaría de Agricultura, Ganadería, Desarrollo Rural, Pesca y Alimentación-Servicio de Información Agroalimentaria y Pesquera). 2006. Situación actual y perspectivas del frijol en México 2000-2005. http:// www.campomexicano.gob.mx/portal_siap/Integracion/EstadisticaDerivada/ComercioExterior/Estudios/Perspectivas/ Frijol00-05.pdf (consultado sept. 2015).

San-Juan-Mejía, Z.M., M.A. Martínez-Damián, y R. García-Mata. 2007. Efecto de las importaciones de carne de cerdo sobre el mercado de carne de res en México. Agrociencia 41:929-938.

SEDAGRO (Secretaría de Desarrollo Agropecuario). 2012. Plan rector sistema producto estatal maíz. http://dev.pue.itesm.mx/ sagarpa/estatales/EPT\%20COMITE\%20SISTEMA\%20PRODUCTO\%20MAIZ\%20EDO\%20DE\%20MEX/PLAN\%20 RECTOR\%20QUE\%20CONTIENE\%20PROGRAMA\%20DE\%20TRABAJO\%202012/PR_MAIZ_MEX_2012.pdf. (consultado sept. 2015).

Serrano, L.M. 2004. Análisis del caso frijol. Universidad Autónoma Chapingo y Secretaría de Economía, MEX.

Turrent, F.A. 2009. Potencial productivo de maíz. Rev. Ciencias 92-93:126-129.

Turrent, F.A., W. Timothy, y G. Elise. 2012. Factibilidad de alcanzar el potencial productivo de maíz de México. GDAE Working Paper No. 12-03. Woodrow Wilson International Center for Scholars, WA, USA.

USDA-ERS (United States Department of Agriculture-Economic Research Services). 2006. Dry beans: policy. https://www.ers. usda.gov/topics/crops/vegetables-pulses/dry-beans/ (accessed sept. 2015). 